ARTICLE

https://doi.org/10.1038/s41467-019-12903-1

\title{
Photoinduced hydrogen release from hydrogen boride sheets
}

\author{
Reiya Kawamura ${ }^{1,8}$, Nguyen Thanh Cuong ${ }^{2,8}$, Takeshi Fujita ${ }^{3}{ }^{3}$, Ryota Ishibiki ${ }^{4}$, Toru Hirabayashi ${ }^{1}$, \\ Akira Yamaguchi ${ }^{1}$, Iwao Matsuda ${ }^{5}$, Susumu Okada ${ }^{2}$, Takahiro Kondo (D) ${ }^{6,7 \star} \&$ Masahiro Miyauchi (i) ${ }^{1 \star}$
}

Hydrogen boride nanosheets ( $\mathrm{HB}$ sheets) are facilely synthesized via ion-exchange treatment on magnesium diboride $\left(\mathrm{MgB}_{2}\right)$ in an acetonitrile solution. Optical absorption and fluorescence spectra of $\mathrm{HB}$ sheets indicate that their bandgap energy is $2.8 \mathrm{eV}$. According to firstprinciples calculations, optical absorption seen at $2.8 \mathrm{eV}$ is assigned to the electron transition between the $\sigma$-bonding states of $\mathrm{B}$ and $\mathrm{H}$ orbitals. In addition, density functional theory (DFT) calculations suggest the other allowed transition from the $\sigma$-bonding state of $\mathrm{B}$ and $\mathrm{H}$ orbitals to the antibonding state with the gap of $3.8 \mathrm{eV}$. Significant gaseous $\mathrm{H}_{2}$ release is found to occur only under photoirradiation, which causes the electron transition from the $\sigma$-bonding state to the antibonding state even under mild ambient conditions. The amount of $\mathrm{H}_{2}$ released from the irradiated $\mathrm{HB}$ sheets is estimated to be $8 \mathrm{wt} \%$, indicating that the sheets have a high $\mathrm{H}_{2}$-storage capacity compared with previously reported metal $\mathrm{H}_{2}$-storage materials.

\footnotetext{
${ }^{1}$ Department of Materials Science and Engineering, Tokyo Institute of Technology, Tokyo 152-8552, Japan. ${ }^{2}$ Department of Physics, Faculty of Pure and Applied Sciences, University of Tsukuba, Tsukuba, Ibaraki 305-8571, Japan. ${ }^{3}$ School of Environmental Science and Engineering, Kochi University of Technology, Kochi 782-8502, Japan. ${ }^{4}$ Graduate School of Pure and Applied Sciences, University of Tsukuba, Tsukuba 305-8573, Japan. ${ }^{5}$ Institute for Solid State Physics, University of Tokyo, Kashiwa, Chiba 277-8581, Japan. ${ }^{6}$ Department of Materials Science and Tsukuba Research Center for Energy Materials Science, Faculty of Pure and Applied Sciences, University of Tsukuba, Tsukuba 305-8573, Japan. ${ }^{7}$ Materials Research Center for Element Strategy, Tokyo Institute of Technology, Yokohama 226-8503, Japan. ${ }^{8}$ These authors equally contributed to this work: Reiya Kawamura, Nguyen Thanh Cuong.

*email: takahiro@ims.tsukuba.ac.jp; mmiyauchi@ceram.titech.ac.jp
} 
$\mathrm{N}$ ew classes of two-dimensional (2D) materials are the subject of considerable research and development efforts for functional applications, including electronics, energy, environment, bioscience, and catalysis, similar to graphene $\mathrm{e}^{1-3}$ and chalcogenide nanosheets 4,5 . Among the various candidates as a new class of $2 \mathrm{D}$ nanosheets beyond graphene ${ }^{6}$, borophene, which is composed of $\mathrm{B}$ atoms arranged in a single monoatomic layer, was theoretically predicted to have unique mechanical and electronic properties ${ }^{7}$ and was successfully synthesized on $\mathrm{Ag}$ (111) under ultrahigh-vacuum conditions ${ }^{8}$. Additionally, borophane, i.e. hydrogenated borophene, which is terminated with hydrogen on a hexagonal boron nanosheet, was theoretically predicted to have sufficient $\mathrm{H}_{2}$ storage, unique mechanical and electronic properties ${ }^{9-12}$. To extend the studies on these B-based $2 \mathrm{D}$ nanosheets, the development of a simple and facile fabrication process, e.g., Scotch-tape exfoliation of graphene, is essential ${ }^{1}$. Recently, Jasuja et al. ${ }^{13}$ reported the synthesis of boron-based nanosheets via exfoliation from magnesium diboride $\left(\mathrm{MgB}_{2}\right)$ under ultrasonication in water ${ }^{14}$. However, its chemical composition was quite complicated, because it would be partially oxidized and still contained magnesium ions on its surface ${ }^{13}$. To understand the basic electrical and/or optical properties of this new class of nanosheets, the synthesis of well-defined and highly pure materials is indispensable for the development of various applications. Recently, our group established the synthesis of the hydrogen boride nanosheets (HB sheets) with a well-defined chemical composition using a facile ion-exchange technique in an organic solvent through an exfoliation process ${ }^{15}$. Interestingly, our $\mathrm{HB}$ sheets produced gaseous $\mathrm{H}_{2}$ under heat treatment at $150-1200^{\circ} \mathrm{C}$ and exhibited a semiconductive photoabsorption property ${ }^{15}$. According to previous theoretical and experimental studies, the $\mathrm{HB}$ sheet is a promising material for $\mathrm{H}_{2}$ storage because its gravimetric $\mathrm{H}_{2}$ storage capacity vs. the light boron element is quite high $(8.5 \mathrm{wt} \%)^{9,15}$. The $\mathrm{HB}$ sheet has more advantages than conventional $\mathrm{H}_{2}$-storage systems such as metalbased materials and high-pressure containers owing to its high $\mathrm{H}_{2}$ capacity and safe operation.

Herein, we report efficient $\mathrm{H}_{2}$ release from $\mathrm{HB}$ sheets using photon energy under extra mild conditions, i.e. the ambient room temperature and atmospheric pressure. We calculated the electronic structure of the HB sheets using density functional theory (DFT) and investigated their optical properties to examine the relationship between the electronic structure and the $\mathrm{H}_{2}$-release property.

\section{Results}

Characterization. The $\mathrm{HB}$ sheets were synthesized via a wet chemical exfoliation method. The starting material was magnesium diboride $\left(\mathrm{MgB}_{2}\right)$, in which $\mathrm{Mg}$ ions were intercalated between each $\mathrm{B}$ layer, forming a honeycomb structure with $s p^{2}$ bonds. $\mathrm{MgB}_{2}$ powder and cation-exchange resin were introduced into an acetonitrile solution, followed by stirring, where $\mathrm{Mg}^{2+}$ ions of $\mathrm{MgB}_{2}$ were exchanged with protons from the resin. This procedure was conducted at room temperature and the atmospheric pressure through $\mathrm{N}_{2}$ bubbling. After 3 days of stirring, the remaining $\mathrm{MgB}_{2}$ and resin were removed via filtration. Finally, we could obtain yellow powder of $\mathrm{HB}$ sheets after the filtrate was dried under reduced pressure at $313 \mathrm{~K}$.

A transmission electron microscopy (TEM) image of the HB sheets is shown in Fig. 1a. Thin and wrinkled structures were observed. Electron energy-loss spectroscopy (EELS) revealed only double peaks of $B$, indicating that the $B$ atoms of the $\mathrm{HB}$ sheets retained their planar $s p^{2}$ bonds even after the cation exchange (Fig. 1b) ${ }^{16,17}$. X-ray photoelectron spectroscopy (XPS) revealed that the $\mathrm{B}$ maintained its negative-charge state and that the $\mathrm{Mg}$ signal disappeared after exfoliation (Fig. 1c), suggesting that cations are replaced with protons. Fourier-transform infrared (FT-IR) spectroscopy revealed the formation of $\mathrm{B}-\mathrm{H}$ and $\mathrm{B}-\mathrm{H}-\mathrm{B}$ bands (see Supplementary Fig. 1). The chemical composition of $\mathrm{HB}$ sheets was previously determined by the thermal desorption mass spectroscopy ${ }^{15}$. Specifically, based on the weight of the sample and quantitatively estimated desorbed hydrogen amount (using calibration made by standard sample measurements), we have estimated the ratio as $\mathrm{H}: \mathrm{B}=1: 1$. In this estimation, we corrected the effect of a small amount of impurity (boric acid) using its decomposed product amount of desorbed water at $\sim 400$ $\mathrm{K}$ (mass number 18) with control experiment. Importantly, the $\mathrm{H} / \mathrm{B}$ ratio obtained from $\mathrm{HB}$ samples with different lot with different amount of impurity always shows the same value of $\mathrm{H}: \mathrm{B}=1: 1$ after the correction ${ }^{15}$. Additionally, we obtained an atomic force microscopy (AFM) image of $\mathrm{HB}$ sheets coated on an atomically flat mica substrate. The thickness of the HB sheets was less than a few nanometers, as shown in Fig. 1d, indicating that the $\mathrm{HB}$ sheets had a multi-layered nature rather than a single layer.

Next, we investigated the optical absorption and fluorescence emission of the $\mathrm{HB}$ sheets and the results are shown in Fig. 2. According to the optical absorption spectrum (Fig. 2a), the bandgap energy of $\mathrm{HB}$ sheets was estimated to be $2.8 \mathrm{eV}$. The three-dimensional (3D) fluorescence spectrum is shown in Fig. 2b, and the fluorescence signal excited by $2.8 \mathrm{eV}(\sim 440 \mathrm{~nm})$ was consistent with the absorption spectrum. Noteworthy, the fluorescence emission signal was terminated when the excitation wavelength was shorter than $350 \mathrm{~nm}$. These results indicate that the unoccupied band dispersion of $\mathrm{HB}$ sheets is $0.6 \mathrm{eV}$, which is relatively narrow as compared to conventional semiconductors.

DFT calculation. To understand the optical property of the HB sheets, we performed first-principles DFT calculations on the model of the HB sheet (Fig. 3a) based on our previous characterization using an X-ray pair distribution function analysis together with Fourier-transform infrared reflection spectroscopy, XPS, and ultraviolet absorption spectroscopy ${ }^{15}$. The calculated model in the present study is consistent with the previous works ${ }^{15,18}$. The calculation was performed based on the DFT framework ${ }^{19,20}$ using the Quantum Espresso code ${ }^{21,22}$ with the semi-local Perdew, Burke, and Ernzerhof (PBE) exchangecorrelation functional ${ }^{23}$. The norm-conserving SG15 pseudopotentials ${ }^{24}$ were used to describe the electron-ion interaction for $\mathrm{B}$ and $\mathrm{H}$ elements and the Brillouin-zone integration was sampled by the Monkhorst-Pack (MP) scheme ${ }^{25}$. The wave functions were expanded using plane-wave basis sets with cutoff energies of 100 Ry $(1360 \mathrm{eV})$ in which the converged geometry and energy-band results were obtained. According to the results for the band structure and state-to-state transition probabilities (Fig. 3b-d, Table 1), the HB sheet has two major transitions, i.e. the $\alpha \rightarrow \beta$ transition $(2.42 \mathrm{eV})$ and the $\alpha \rightarrow \gamma$ transition $(3.85 \mathrm{eV})$, as shown in Fig. 3c. The former transition is attributed to the experimentally observed optical absorption. Interestingly, the HB sheet involves optical allowed transition induced by $3.85 \mathrm{eV}$ photon energy $(\sim 320 \mathrm{~nm})$. The a state, which is shown in Fig. 3, is the $\sigma$ bond that consists of B $2 p_{y}$ and $\mathrm{H} 1 s$ atomic orbitals, and the $\gamma$ state is the $\sigma$-antibonding of the $\alpha$-state. In contrast, the origin of the $\beta$ state is the $\sigma$-bond of $\mathrm{B} 2 p_{x}$ atomic orbitals. The expected probabilities of each transition calculated via DFT are shown in Table 1. Semi-local functionals such as PBE often underestimate the bandgap ${ }^{26}$. Thus, we performed energy-band calculations of the HB monolayer with different versions of hybrid Heyd-Scuseria-Ernzerhof (HSE) and B3LYP exchangecorrelation functionals, as shown in the Supplementary 
a

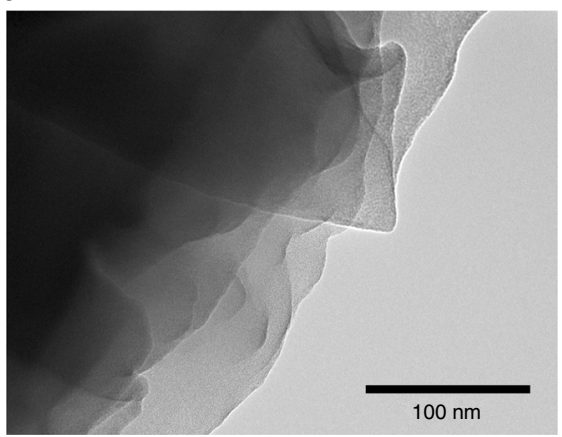

C

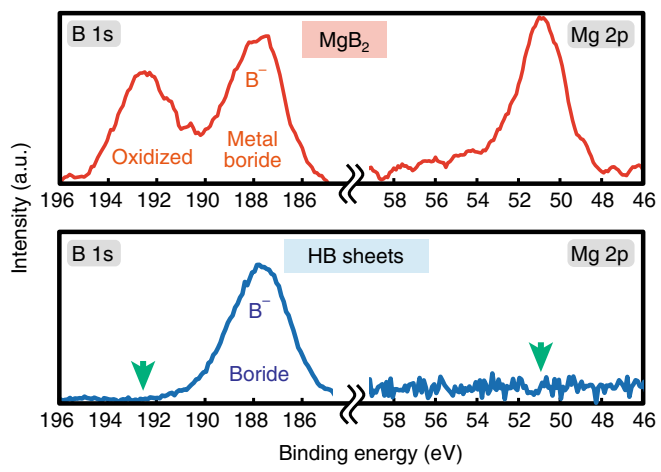

b

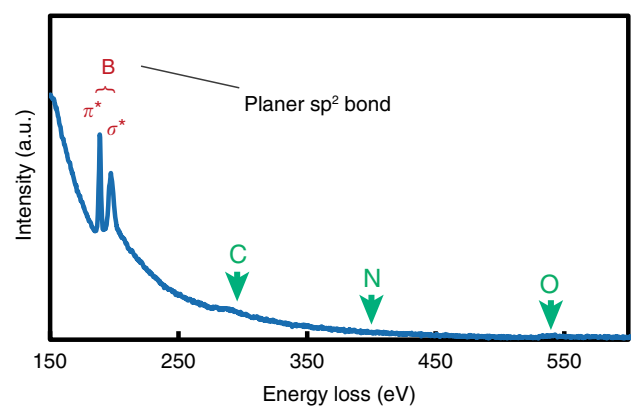

d

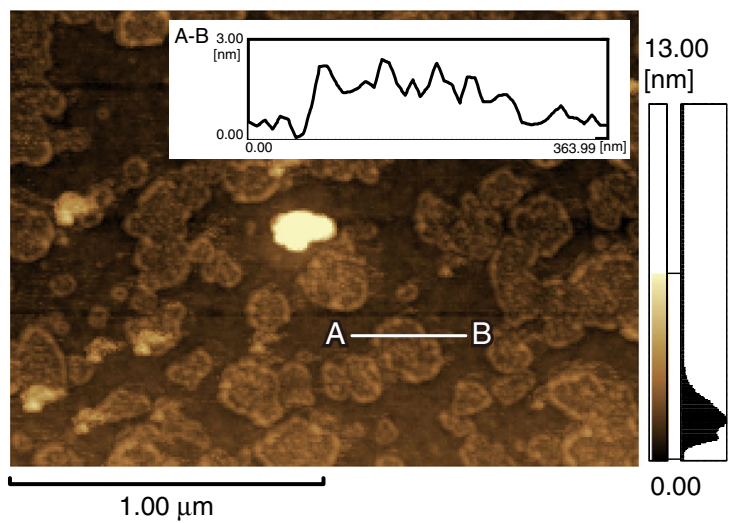

Fig. 1 Characterization of HB sheets. a TEM image and $\mathbf{b}$ electron energy loss spectroscopy (EELS) of HB sheets, $\mathbf{c} X P S$ results for MgB 2 and $H B$ sheets, and d AFM image of HB sheets on mica substrate and line profile between points $A$ and $B$ in AFM image
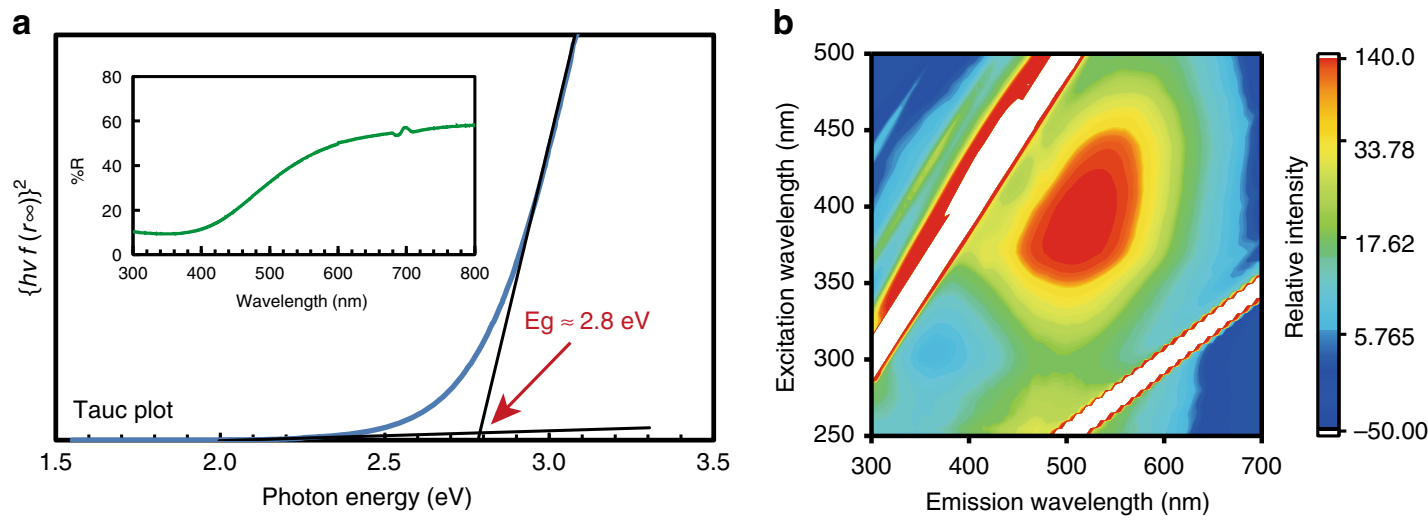

Fig. 2 Optical properties of HB sheets. a Absorption and $\mathbf{b}$ 3D fluorescence spectra of HB sheets

Information (Table 1). The transition energies (energy gap) between $\alpha$-, $\beta$-, and $\gamma$-states at the $\Gamma$-point of the HB monolayer increased significantly from 2.4 and $3.8 \mathrm{eV}$ of the PBE functionals to 2.69 (2.54) and $4.5(4.93) \mathrm{eV}$ of the HSE03 (B3LYP) functionals, respectively. However, it was reported that the hybrid exchange-correlation potentials such as HSE and B3LYP do not accurately describe electronic properties such as $\mathrm{MgB}_{2}{ }^{18}$. Moreover, the present calculated result for the $\mathrm{HB}$ sheet using $\mathrm{PBE}$ functionals is consistent with that of a recent report ${ }^{27}$. Furthermore, we calculated the bilayer structures of the $\mathrm{HB}$ sheet since the AFM image indicated that the HB sheets existed had a multilayered rather than a mono-layered nature. Supplementary Fig. 2 shows top and side views of optimized atomic structures, the energy band of the HB monolayer, and four possible stacking HB bilayers. Consequently, the HB layer is weakly bound together via the van der Waals interaction, indicating that the electronic property of $\mathrm{HB}$ sheets exhibits like a mono-layered characteristic. These calculation results indicate the HB sheets have unique two band structure.

$\mathbf{H}_{2}$ release property. Next, we investigated the $\mathrm{H}_{2}$-release property of the HB sheets in a flow-type reactor through the excitation corresponding to the two bands that indicated by DFT calculations (See Supplementary Fig. 3). A mercury-xenon lamp (200 W $\mathrm{Hg}$-Xe lamp as a UV light) induced the $3.8 \mathrm{eV}$ transition, while a xenon lamp (150 W Xe lamp as a visible light) induced the $2.8 \mathrm{eV}$ transition. Further, we used a cutoff filter (Y-490, cutoff below $490 \mathrm{~nm}$ ) with these light sources, which led to excitation of neither transition, as a control. Figure 4a shows the results for $\mathrm{H}_{2}$ 
a

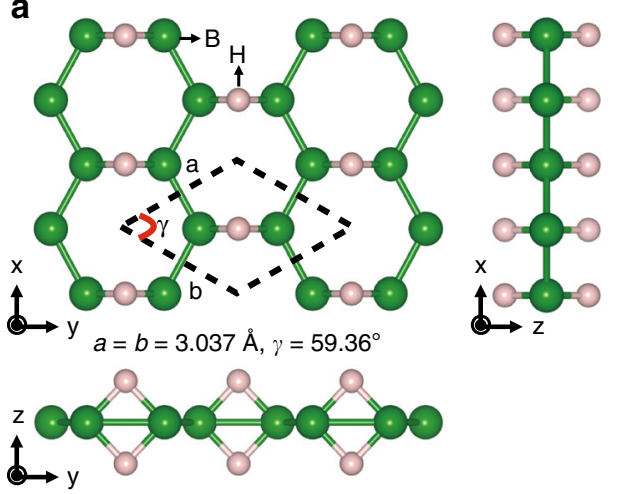

C

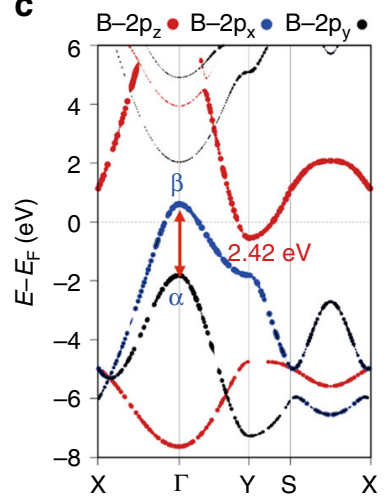

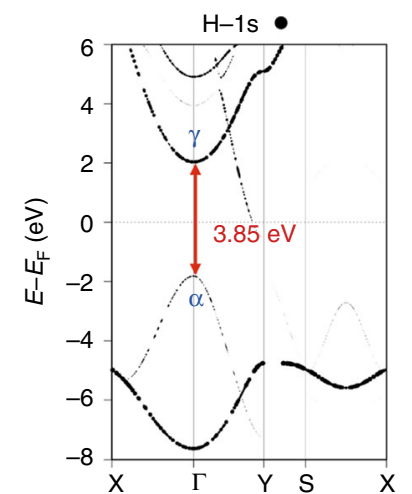

b

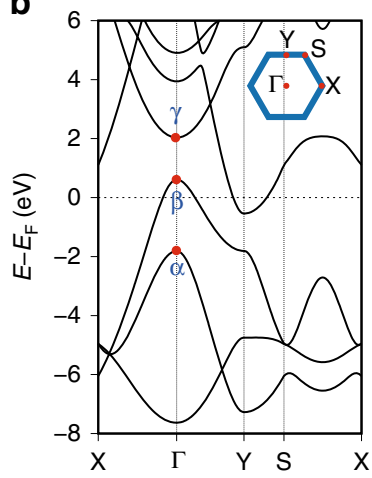

d

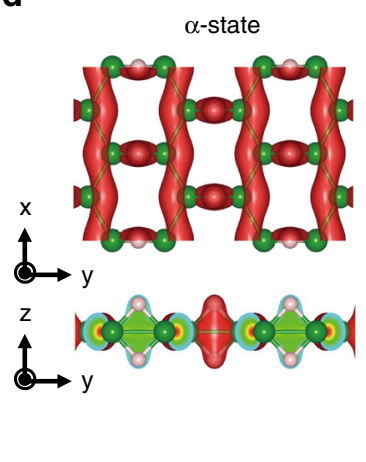

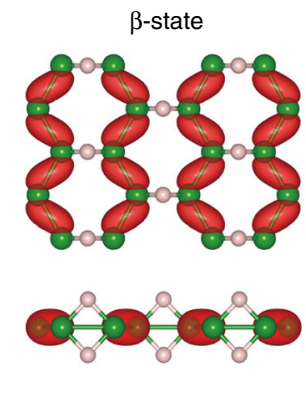

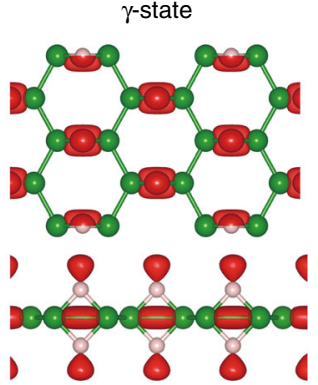

Fig. 3 First-principles calculations for an HB sheet. a Top and side views of the optimized atomic structure of the HB sheet. The bonds drawings are defined by the interatomic distance, and the unit cell is indicated by the dashed lines. $\mathbf{b}$ Electronic band structure of the HB sheet and the Brillouin zone. $\mathbf{c}$ Projected band structures of B- and H-originated orbitals. $\mathbf{d}$ Square-wave function distribution of the $\alpha, \beta$, and $\gamma$ states at the $\Gamma$-point in panel $\mathbf{b}$

\begin{tabular}{|c|c|c|c|c|}
\hline Transition & $\Delta E$ & $\boldsymbol{P}_{\boldsymbol{x}}$ & $\boldsymbol{P}_{\boldsymbol{y}}$ & $P_{z}$ \\
\hline$\alpha \rightarrow \beta$ & $2.42 \mathrm{eV}$ & 0.499 & $1.60 \times 10^{-5}$ & $1.11 \times 10^{-5}$ \\
\hline$\alpha \rightarrow \gamma$ & $3.85 \mathrm{eV}$ & $1.45 \times 10^{-5}$ & 0.464 & 0.036 \\
\hline
\end{tabular}

production under the visible light and UV light with and without the cutoff filter. Significant release of $\mathrm{H}_{2}$ gas from the HB sheets only occurred under the UV light ( $\mathrm{Hg}-\mathrm{Xe}$ lamp) without the cutoff filter, which induced the $a \rightarrow \gamma$ transition. On the other hand, $\mathrm{H}_{2}$ was not released under visible-light irradiation using the Xe lamp, although the HB sheets absorbed the visible light. These results strongly indicate that the $\mathrm{H}_{2}$ release is driven by the $\alpha \rightarrow \gamma$ transition rather than $\alpha \rightarrow \beta$. The $\mathrm{H}_{2}$ release exhibited a rapid response to the on-off procedure of light irradiation, excluding the contribution of the heat effect. Our experimental and theoretical investigations suggest that $\mathrm{H}_{2}$ release is driven by the excited electrons in the antibonding state of the $\mathrm{H}$ orbital, causing the self-reduction of protons and the generation of $\mathrm{H}_{2}$, as shown in Supplemental information (see Supplementary Fig. 4). According to the theoretical calculation by Abtew et al. ${ }^{9}$ the hydrogen bonding strength with boron would be weakened by the electron doping into the antibonding state of $\mathrm{H}$ orbital in hydrogenated borophene, which is consistent with our experimental and theoretical results in terms of the relationship between the electron filling at the antibonding states and the bond strength. The excited electrons in the antibonding state of HB sheets are used for hydrogen production, thus the fluorescence emission by the recombination of electron-hole pairs between a and $\gamma$ states is not observed in Fig. 2b. The previous studies reported multi-fluorescence emissions from boron-based nanosheets ${ }^{28,29}$, indicating that the photo-emission property from HB sheets would strongly depend on defects and/or surface functionalization. In addition, we checked the production of gasphase boron species by mass spectroscopic analysis (see Supplementary Fig. 5). The formation of gas-phase boron species such as $\mathrm{BH}_{3}$ and $\mathrm{B}_{2} \mathrm{H}_{6}$ was found to be negligible or slightly seen at the beginning of light irradiation. We could temporarily detect the water and acetonitrile at the beginning of the light irradiation owing to the desorption of these species, but $\mathrm{H}_{2}$ was continuously evolved.

To investigate the amount of $\mathrm{H}_{2}$ released with respect to the unit weight of the $\mathrm{HB}$ sheets, we used a closed reactor, as shown in Fig. 4 b. The concentration of $\mathrm{H}_{2}$ became saturated around 7.5 $\mu \mathrm{mol}$, corresponding to $8 \mathrm{wt} \%$ of the $\mathrm{HB}$ sheets, by considering the optical light penetration depth in the HB sheets $(\sim 13 \mu \mathrm{m}$ as explained in the Experimental section). The $\mathrm{H}_{2}$ capacity of a unit cell of the HB sheets was $8.5 \mathrm{wt} \%$, thus the amount of $\mathrm{H}_{2}$ released from the HB sheets was close to the theoretical storage capacity. It is noteworthy that the almost all of the terminated $\mathrm{H}$ ions of the $\mathrm{HB}$ sheets were reduced to $\mathrm{H}_{2}$ molecules under photon irradiation under extra mild conditions. The HB sheets consist of a few nanometers ultrathin structure, thus, almost all of the surface $\mathrm{H}$ ions are photoactive species for $\mathrm{H}_{2}$ production. This is a significant advantage compared with $\mathrm{H}_{2}$ storable bulk metal alloys, whose $\mathrm{H}_{2}$ capacities are less than a few mass percent versus the bulk alloy ${ }^{30}$. Conventional $\mathrm{H}_{2}$-storage materials are composed of metal, whose Fermi level is located in an occupied band; thus, photoinduced $\mathrm{H}_{2}$ release has never been observed in these bulk metal materials. It is noteworthy that the $\mathrm{HB}$ sheets exhibit a 
a
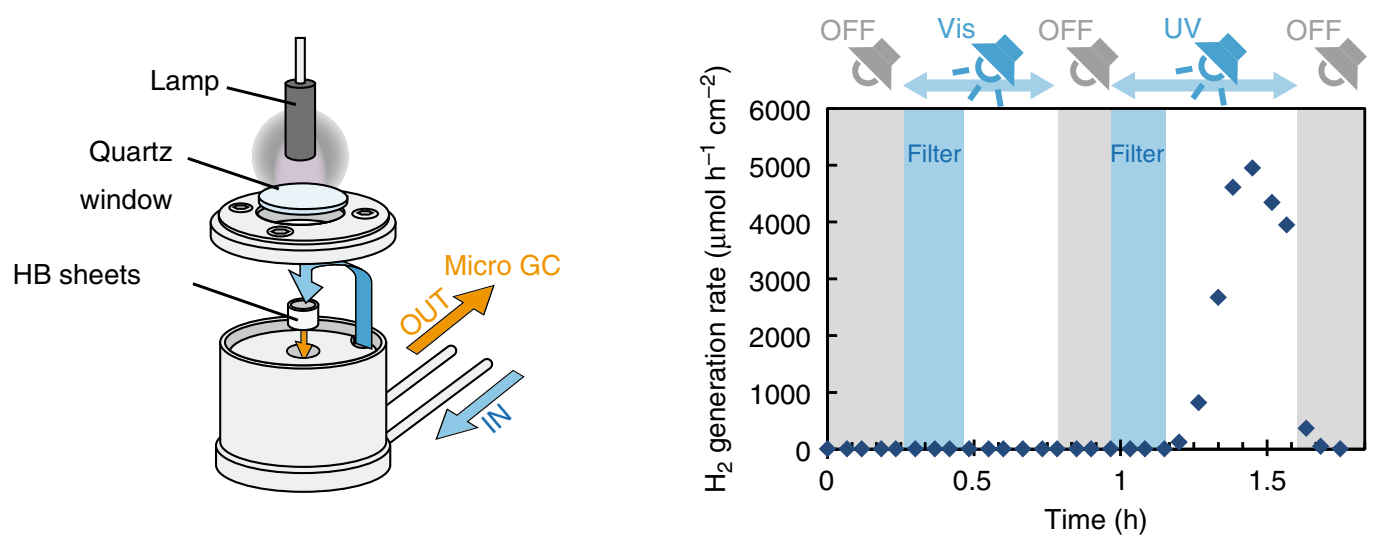

b
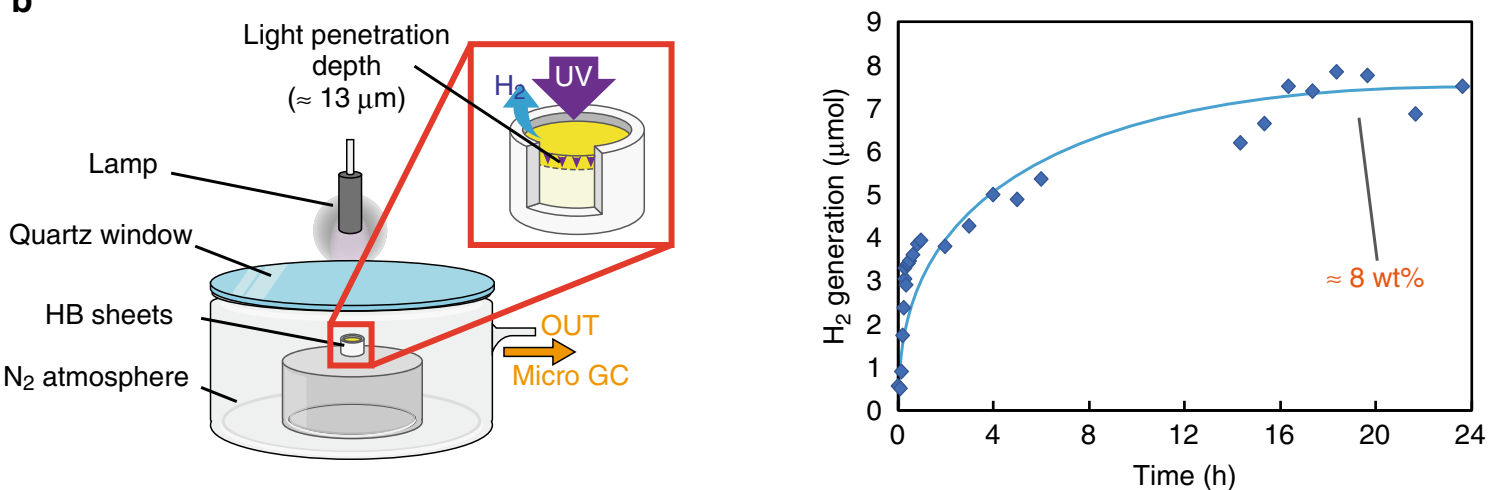

C
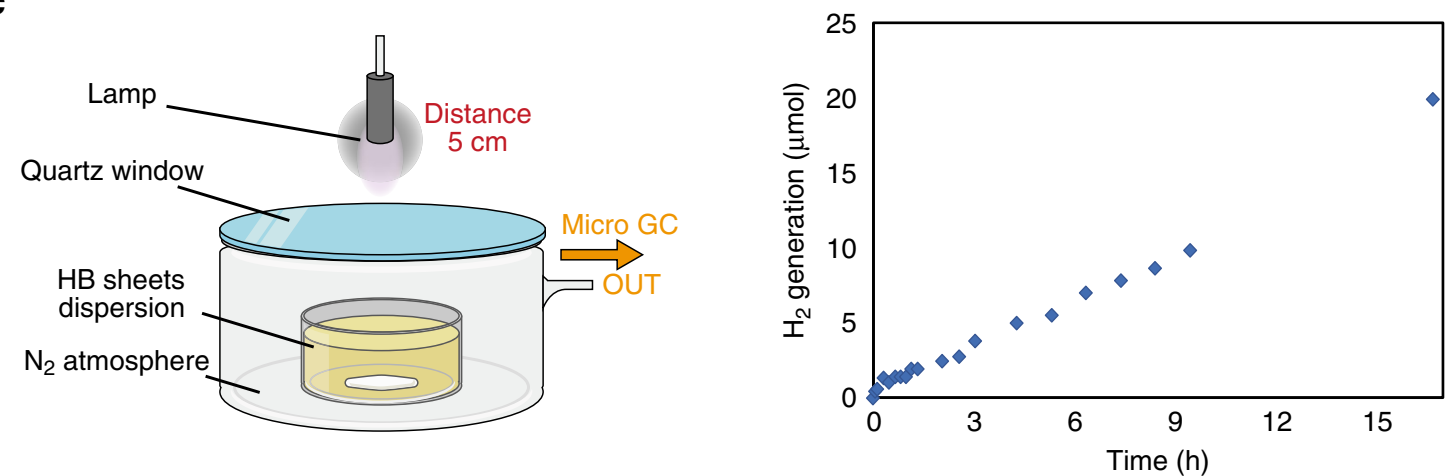

d

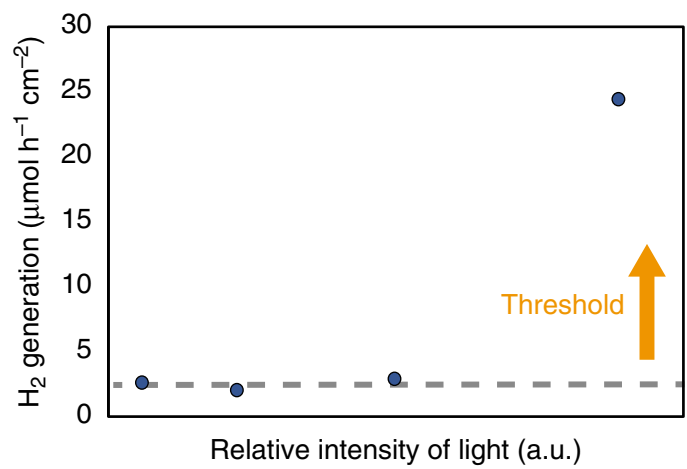

Fig. 4 Photoinduced $\mathrm{H}_{2}$ release properties of $\mathrm{HB}$ sheets. a Schematic illustrations of the experimental setup and $\mathrm{H}_{2}$ production under different light sources in a flow reactor; $\mathbf{b}$ amount of $\mathrm{H}_{2}$ produced under UV irradiation, where the corresponding amount of $\mathrm{HB}$ sheets was estimated by the light penetration depth in $\mathrm{HB}$ sheets; $\mathbf{c} \mathrm{H}_{2}$ production under UV light from $\mathrm{HB}$ sheets dispersed in acetonitrile; d light intensity dependence on $\mathrm{H}_{2}$ production from dispersed $\mathrm{HB}$ sheets, respectively. Each plot in panel $\mathbf{d}$ was measured under the distance between light source and sample at 20, 10, 6.6, 5.0 cm, respectively. Light irradiation condition in panels $\mathbf{b}$ and $\mathbf{c}$ was $5 \mathrm{~cm}$ distance between light source and sample 
semiconductive light absorption property and a high $\mathrm{H}_{2}$ capacity, because $\mathrm{H}$ ions are terminated on the surface of the nanosheet. Therefore, we expect that $\mathrm{HB}$ sheets can be used for safe, lightweight, and photoresponsive $\mathrm{H}_{2}$ carriers.

Our HB sheets could be highly dispersed in a solvent such as alcohol or acetonitrile, thus, we investigated the $\mathrm{H}_{2}$-release property of $\mathrm{HB}$ sheets dispersed in acetonitrile (Fig. 4c). $\mathrm{H}_{2}$ was released at a constant rate under light irradiation even with the dispersion system. This indicates that our $\mathrm{HB}$ sheets can be utilized as $\mathrm{H}_{2}$ carriers in liquid-phase systems in addition to gasphase systems, which enables great advantage for practical use. Using this liquid system setup, we can exclude the photothermal effect on the hydrogen release property since the boiling point of acetonitrile is $82^{\circ} \mathrm{C}$. The actual temperature of acetonitrile under UV light irradiation was $28^{\circ} \mathrm{C}$, implying that the hydrogen evolution from $\mathrm{HB}$ sheets was driven through the electron transition by photons rather than heat generation. Further, we investigated the light intensity dependence on the $\mathrm{H}_{2}$-release property for the liquid-phase dispersed system by changing the distance between the light source and the samples (Fig. $4 \mathrm{~d}$ ). The $\mathrm{H}_{2}$ molecules were released under certain photon-flux threshold, indicating that the density of electron-hole pairs is important for driving hydrogen evolution. This phenomenon differs from a conventional photochemical reaction under a lightlimited condition in conventional photocatalysis.

Our experimental and theoretical studies in the present work strongly suggested that the excited electrons at the antibonding sites of $\mathrm{H}$ play an important role in the production of $\mathrm{H}_{2}$ from $\mathrm{HB}$ sheets. In addition to the reduction reaction caused by excited electrons, we discuss the photogenerated holes in the bonding state of the B orbital under UV irradiation. The generated holes in the $\mathrm{B}$ orbital oxidize negatively charged $\mathrm{B}$ to an intermediate lesscharged state. We attempted to detect the intermediate state of B under photoirradiation in a sample-exchange pre-chamber equipped with an XPS apparatus through a quartz window (see Supplementary Fig. 6a). However, the HB sheets were easily oxidized by $\mathrm{O}$ contaminants, resulting in a positive-charge state of B (Supplementary Fig. 6b), because of the insufficient vacuum level $\left(\sim 10^{-4} \mathrm{~Pa}\right)$ in the pre-chamber. However, interestingly, we detected the intermediate charged state of $\mathrm{B}\left(\mathrm{B}_{\text {in }}\right)$ between $\mathrm{B}^{+}$and $\mathrm{B}^{-}$signals in an ultrahigh-vacuum chamber $\left(\sim 10^{-8} \mathrm{~Pa}\right)$ under low-energy X-ray exposure (see Supplementary Fig. 6d). We consider that the intermediate charged $\mathrm{B}$ states were temporarily formed under UV irradiation, leading to $\mathrm{H}_{2}$ generation, similar to the case of heat treatment. With UV irradiation under the ambient atmospheric conditions, amorphous or partially oxidized $B$ would be produced after the release of $\mathrm{H}_{2}$. The FT-IR spectra and TEM image of HB sheets after UV light irradiation are shown in the Supplementary Information (see Supplementary Figs. 7 and $8)$. The B-H bonding signal $\left(\sim 2500 \mathrm{~cm}^{-1}\right)$ decreased significantly after light irradiation, while their sheet-like morphology was observed even after UV irradiation.

We note here that the boric acid was sometimes included in the pure HB sheets because of the incomplete separation of boric acid from HB during synthesis (e.g. Supplementary Fig. 1). To check the effect of these included boric acid on the light-induced hydrogen production, we did control experiment, i.e. we examined optical properties (absorption and fluorescence) and hydrogen release property of commercial boric acid. We confirmed that the boric acid is an ultra-widegap material and did not overlap the optical property of $\mathrm{HB}$ sheets. Further, no hydrogen was released from the boric acid under the same UV irradiation. Thus, we can safely exclude the effect of boric acid on the light-induced hydrogen production from HB sheets. Furthermore, the effect of water molecules on the hydrogen generation was investigated. We added HB sheets into water and evaluated their hydrogen production property (see Supplementary Fig. 9). Although the small amount of hydrogen (2.3\% of HB molecules) was released through hydrolysis reaction, significant $\mathrm{H}_{2}$ release under UV irradiation cannot be simply explained by hydrolysis. Other data, including DFT calculations, light wavelength, and intensity dependence, support that the $\mathrm{H}_{2}$ molecules are released from $\mathrm{HB}$ sheets by photogenerated charge carriers, rather than by hydrolysis reaction or decomposition of boric acid impurities.

It is noted that the amount of released hydrogen from the irradiated $\mathrm{HB}$ sheets was $8 \mathrm{wt} \%$. This indicates that the unique optical and physical properties presented in this study are owing to the HB sheet characteristics rather than boric acid or adsorbed water. Re-storage of hydrogen into $\mathrm{HB}$ sheets is one of the most significant challenges for practical application as $\mathrm{H}_{2}$ carriers. However, we expect the $\mathrm{HB}$ sheets to have the rechargeable $\mathrm{H}_{2}$ property, because of the capability of $\mathrm{H}_{2}$ to absorb amorphous $\mathrm{B}^{31}$. The $\mathrm{H}_{2}$ re-storage property of $\mathrm{HB}$ sheets is currently under investigation and will be reported elsewhere.

\section{Discussion}

In the present study, significant $\mathrm{H}_{2}$ release was induced from $\mathrm{HB}$ sheets only by photon irradiation. Our HB sheets are lightweight, and their $\mathrm{H}_{2}$-storage capacity is higher than that of bulk alloys. The significant $\mathrm{H}_{2}$ release from the $\mathrm{HB}$ sheets was due to their $2 \mathrm{D}$ nanosheet structure and unique electronic band structure. This intriguing phenomenon enables us to provide a portable hydrogen generator wherever on-site using $\mathrm{HB}$ sheets and a light source. The results also indicate that the photogenerated charge carriers in $\mathrm{HB}$ sheets can initiate a chemical reaction, in addition to $\mathrm{H}_{2}$ production. We expect the present study to stimulate research on hydrogen boride for various fields not only for $\mathrm{H}_{2}$ storage applications but also for electrical and semiconductive materials, electrochemical devices, photocatalysts, etc.

\section{Methods}

Synthesis of HB sheets. Magnesium diboride powder $\left(\mathrm{MgB}_{2}, 500 \mathrm{mg}, 99 \%\right.$; Sigma-Aldrich, St. Louis, MO, USA) and a cation-exchange resin $(30 \mathrm{~mL}$ Amberlite IR120B hydrogen form; Organo Corp., Tokyo, Japan) were added to acetonitrile (99.5\%, JIS special grade; Wako, Osaka, Japan) at room temperature, followed by stirring at the atmospheric pressure in an $\mathrm{N}_{2}$ atmosphere. After 3 days of stirring, the remaining $\mathrm{MgB}_{2}$ and resin were removed from the solution via a membrane filter (Omnipore $0.2 \mu \mathrm{m}$; Merck Millipore Ltd, Ireland). The filtrate, which was a yellow dispersion, was dried under a reduced pressure at $313 \mathrm{~K}$, yielding $\mathrm{HB}$ sheets in the form of yellow powder.

Characterization. Transmission electron microscopic (TEM) was performed using JEM-2100F TEM/STEM (JEOL Ltd, Japan) operated at an acceleration electronbeam voltage of $200 \mathrm{kV}$. XPS measurement was performed using JPS $9010 \mathrm{TR}$ (JEOL, Ltd, Japan) with an Al Ka X-ray source $(1486.6 \mathrm{eV})$. The samples were fixed on graphite tape. The absolute binding energy was calibrated according to the peaks of $\mathrm{B} 1 s$ in $\mathrm{B}(\mathrm{OH})_{3}$ or the $\mathrm{C} 1 s$ orbitals. Fourier-transform infrared spectroscopy (FT-IR) spectra were recorded using FT/IR-6100 (JASCO, Co., Ltd, Japan). Approximately $0.25 \mathrm{wt} \%$ of $\mathrm{HB}$ sheets was mixed with $\mathrm{KBr}$ powder to fabricate pellets for FT-IR measurement. UV-visible (UV-vis) diffuse reflectance spectra were recorded using a spectrophotometer (V-670, JASCO, Co., Ltd, Japan) equipped with an integration sphere unit. The bandgap of the HB sheets was calculated via extrapolation in the Tauc-plot curve of the Kubelka-Munk function. A white $\mathrm{BaSO}_{4}$ plate was used as a reflectance standard. The $3 \mathrm{D}$ fluorescence spectra of the HB sheets were recorded using a spectrofluorophotometer (F-7000; Hitachi High-Tech Science Corporation, Ltd, Japan). The measurement was conducted at room temperature in an $\mathrm{N}_{2}$ atmosphere. For an AFM measurement, the $\mathrm{HB}$ sheets were coated on an atomically flat mica substrate via spin coating using the solution of HB sheets dispersed in acetonitrile. The spin coating was conducted at 2000 r.p.m. for $20 \mathrm{~s}$. AFM images were obtained in the dynamic mode using SPM-9700 (Shimadzu Corp., Kyoto, Japan) with a silicon cantilever.

DFT calculation. First-principles total-energy calculations were performed within the framework of DFT ${ }^{19,20}$ by using the Quantum Espresso code ${ }^{21,22}$ with the Perdew, Burke, and Ernzerhof exchange-correlation functional ${ }^{23}$. The normconserving SG15 pseudopotentials ${ }^{24}$ were used to describe the electron-ion interaction for $\mathrm{B}$ and $\mathrm{H}$ elements. The valence wave functions were expanded using a plane-wave basis set with a cutoff energy of $100 \mathrm{Ry}$. Both the internal atomic 
coordinates and lattice parameters of borophane monolayer were fully optimized until the residual forces acting on each atom were less than $10^{-4} \mathrm{Ry} / \mathrm{Bohr}$. The $1 \times 1$ unit cell was used for the borophane monolayer. To simulate the isolated borophane monolayer, the sheet was separated by periodic images with a vacuum region of $10 \AA$. The Brillouin-zone integration was sampled by the Monkhorst-Pack (MP) scheme ${ }^{25}$ with $42 \times 42 \times 1$ k-point grids in the selfconsistent field calculations for optimization structures and energy band structures. The state-to-state transition probabilities were estimated according to the transition dipole moment from state $i$ to state $j$ and were normalized by a factor of $10^{5}$. The exciton effect is significant in low-dimensional systems because electrostatic screening is limited. The relatively good agreement of the experimental fluorescence energies and simulation energy gaps is thus considered to be due to the error cancelation between the underestimation of the fundamental bandgap and the absence of the exciton effect in our DFT calculations. All calculations were performed on the SGI ICE XA/UV supercomputer at the Institute of Solid State Physics, The University of Tokyo, Japan.

$\mathbf{H}_{\mathbf{2}}$-release property of $\mathbf{H B}$ sheets. To evaluate the photoinduced $\mathrm{H}_{2}$-release property of the $\mathrm{HB}$ sheets, $20 \mathrm{mg}$ of $\mathrm{HB}$ powder was mounted on a porous ceramic cup $(0.48 \mathrm{~cm}$ in diameter), and the cup was placed in a flow reactor, as described in the main text (Fig. 4a). Argon gas was flowed through this reactor $(10 \mathrm{~mL} / \mathrm{min})$ and passed through pores in the ceramic cup, and then the hydrogen evolution at an outlet was measured using a micro-gas chromatograph (micro-GC, 90 Micro GC; Agilent Technologies, Co., Ltd, USA) under light irradiation. Inner diameter of the ceramic cup was $0.48 \mathrm{~cm}$, and irradiated area was $0.18 \mathrm{~cm}^{2}$. We used two types of light sources (Supplementary Fig. 3 ) and a cutoff filter (Y-49) which cannot transmit light with a wavelength shorter than $490 \mathrm{~nm}$. We also measured the photoinduced $\mathrm{H}_{2}$ release from an HB sheet dispersion. HB sheets were dispersed in acetonitrile in a glass beaker, which was placed in a 500-mL closed glass cell filled with inert gas, as shown in Fig. 4c. Light irradiation was performed through a quartz window of the cell, and then the $\mathrm{H}_{2}$ concentration was measured using the micro-GC ( 90 Micro GC; Agilent Technologies, Co., Ltd, US). We investigated the light intensity dependence on $\mathrm{H}_{2}$ release by changing the distance between the light source and the dispersed samples (Fig. 4d).

\section{Estimation of light penetration depth for determination of released $\mathbf{H}_{2}$} amount vs. unit mass of HB sheets. To estimate the light penetration depth into the HB sheets, we fabricated a thin film of HB sheets on a transparent quartz substrate. The thickness and transmittance of this film were measured using a three-dimensional laser microscope (LEXT OLS4000; Olympus, Co., Ltd, Japan) and a spectrophotometer (V-670; JASCO, Co., Ltd, Japan), respectively. Based on these results, the absorption coefficient $(\alpha)$ was estimated according to the Beer-Lambert law. On the basis of the $\alpha$ value, we roughly estimated the light penetration depth as the distance where the light intensity was $1 \%$ that of the incident light at $360 \mathrm{~nm}$ because the photon density was the highest around $360 \mathrm{~nm}$ for the Hg-Xe lamp used in the present study. Under this condition, the light penetration depth was $13 \mu \mathrm{m}$. The amount of irradiated HB sheets was calculated according to the light penetration depth and the packing density of the HB sheets in a ceramic cup. The results indicated that $8 \mathrm{wt} \%$ of $\mathrm{H}_{2}$ was released from the irradiated $\mathrm{HB}$ sheets.

\section{Data availability}

The data that support the findings of this study are available from the article and Supplementary Information files, or from the corresponding authors upon reasonable request.

Received: 20 March 2019; Accepted: 7 October 2019; Published online: 25 October 2019

\section{References}

1. Novoselov, K. S. et al. Electric field effect in atomically thin carbon films. Science 306, 666-669 (2004).

2. Eda, G. \& Chhowalla, M. Chemically derived graphene oxide: towards largearea thin-film electronics and optoelectronics. Adv. Mater. 22, 2392-2415 (2010).

3. Soy, E., Guisinger, N. P. \& Trenary, M. Growth of Pd nanoclusters on singlelayer graphene on $\mathrm{Cu}(111)$. J. Phys. Chem. B 122, 572-577 (2018).

4. Chhowalla, M. et al. The chemistry of two-dimensional layered transition metal dichalcogenide nanosheets. Nat. Chem. 5, 263 (2013).

5. Ma, R. \& Sasaki, T. Nanosheets of oxides and hydroxides: ultimate 2D chargebearing functional crystallites. Adv. Mater. 22, 5082-5104 (2010).

6. Kong, X., Liu, Q., Zhang, C., Peng, Z. \& Chen, Q. Elemental two-dimensional nanosheets beyond graphene. Chem. Soc. Rev. 46, 2127-2157 (2017).
7. Kunstmann, J. \& Quandt, A. Broad boron sheets and boron nanotubes: an ab initio study of structural, electronic, and mechanical properties. Phys. Rev. B 74, 035413 (2006)

8. Mannix, A. J. et al. Synthesis of borophenes: anisotropic, two-dimensional boron polymorphs. Science 350, 1513-1516 (2015).

9. Abtew, T. A., Shih B.-C, Dev, P., Crespi, V. H. \& Zhang, P. Prediction of a multicenter-bonded solid boron hydride for hydrogen storage. Phys. Rev. B $\mathbf{8 3}$, 094108 (2011).

10. Jiao, Y., Ma, F., Bell, J., Bilic, A. \& Du, A. Two-dimensional boron hydride sheets: high stability, massless dirac fermions, and excellent mechanical properties. Angew. Chem. Int. Ed. 55, 10292-10295 (2016).

11. Mortazavi, B. et al. Borophene hydride: a stiff $2 \mathrm{D}$ material with high thermal conductivity and attractive optical and electronic properties. Nanoscale 10, 3759-3768 (2018).

12. Xu, L.-C., Du, A. \& Kou, L. Hydrogenated borophene as a stable twodimensional Dirac material with an ultrahigh Fermi velocity. Phys. Chem. Chem. Phys. 18, 27284-27289 (2016).

13. James, A. L. \& Jasuja, K. Chelation assisted exfoliation of layered borides towards synthesizing boron based nanosheets. RSC Adv. 7, 1905-1914 (2017).

14. Das, S. K. \& Jasuja, K. Chemical exfoliation of layered magnesium diboride to yield functionalized nanosheets and nanoaccordions for potential flame retardant applications. ACS Appl. Nano Mater. 1, 1612-1622 (2018).

15. Nishino, H. et al. Formation and characterization of hydrogen boride sheets derived from MgB2 by cation exchange. J. Am. Chem. Soc. 139, 13761-13769 (2017).

16. Stephan, O. et al. Doping graphitic and carbon nanotube structures with boron and nitrogen. Science 266, 1683-1685 (1994).

17. Kouvetakis, J. et al. Novel aspects of graphite intercalation by fluorine and fluorides and new $\mathrm{B} / \mathrm{C}, \mathrm{C} / \mathrm{N}$ and $\mathrm{B} / \mathrm{C} / \mathrm{N}$ materials based on the graphite network. Synth. Met. 34, 1-7 (1989).

18. Gao, W. et al. On the applicability of hybrid functionals for predicting fundamental properties of metals. Solid State Commun. 234-235, 10-13 (2016).

19. Hohenberg, P. \& Kohn, W. Inhomogeneous electron gas. Phys. Rev. 136, B864-B871 (1964).

20. Kohn, W. \& Sham, L. J. Self-consistent equations including exchange and correlation effects. Phys. Rev. 140, A1133-A1138 (1965).

21. Giannozzi, P. et al. QUANTUM ESPRESSO: a modular and open-source software project for quantum simulations of materials. J. Phys. Condens. Matter 21, 395502 (2009).

22. Giannozzi, P. et al. Advanced capabilities for materials modelling with Quantum ESPRESSO. J. Phys. Condens. Matter 29, 465901 (2017).

23. Perdew, J. P., Burke, K. \& Ernzerhof, M. Generalized gradient approximation made simple. Phys. Rev. Lett. 77, 3865-3868 (1996).

24. Schlipf, M. \& Gygi, F. Optimization algorithm for the generation of ONCV pseudopotentials. Comput. Phys. Commun. 196, 36-44 (2015).

25. Monkhorst, H. J. \& Pack, J. D. Special points for Brillouin-zone integrations. Phys. Rev. B 13, 5188-5192 (1976).

26. Tran, F. \& Blaha, P. Accurate band gaps of semiconductors and insulators with a semilocal exchange-correlation potential. Phys. Rev. Lett. 102, 226401 (2009).

27. Tateishi, I. et al. Semimetallicity of free-standing hydrogenated monolayer boron from $\mathrm{MgB}_{2}$. Phys. Rev. Mater. 3, 024004 (2019).

28. Das, S. K., Bedar, A., Kannan, A. \& Jasuja, K. Aqueous dispersions of fewlayer-thick chemically modified magnesium diboride nanosheets by ultrasonication assisted exfoliation. Sci. Rep. 5, 10522 (2015).

29. John, S.K. \& Anappara, A. A. Aqueous dispersions of highly luminescent boron-rich nanosheets by the exfoliation of polycrystalline titanium diboride. N. J. Chem. 43, 9953-9960 (2019).

30. Sandrock, G. A panoramic overview of hydrogen storage alloys from a gas reaction point of view. J. Alloy Compd. 293-295, 877-888 (1999).

31. Wang, P., Orimo, S. \& Fujii, H. Characterization of hydrogenated amorphous boron by a combination of infrared absorption spectroscopy and thermal analyses. J. Alloy Compd. 359, L1-L3 (2003).

\section{Acknowledgements}

This work is supported by JSPS Kakenhi (Grant No. 18H02055, 18H03874, 19H02551, 19H05046). This work is also supported by MEXT Element Strategy Initiative to Form Core Research Center, Murata Science Foundation, KUMAGAI, Ogasawara, and Samco foundations for the Promotion of Science \& Engineering. We appreciate Ms. M. Koitabashi for her evaluation of hydrogen production property from HB sheets in water.

\section{Author contribution}

R.K. conducted the experimental studies and wrote the paper. N.T.C. and S.O. carried out the DFT calculation and N.T.C. wrote the paper. T.F. performed TEM analysis, and 
R.I., T.H., T.K., and I.W. conducted spectroscopic analysis. A.Y. contributed to the experimental design and gave deep insight into photochemical reaction. T.K. established the synthetic method and wrote the paper. M.M. conceived this project and wrote the paper. All authors discussed the results and commented on the manuscript.

\section{Competing interests}

The authors declare no competing interests.

\section{Additional information}

Supplementary information is available for this paper at https://doi.org/10.1038/s41467019-12903-1.

Correspondence and requests for materials should be addressed to T.K. or M.M.

Peer review information Nature Communications thanks the anonymous reviewers for their contributions to the peer review of this work.

Reprints and permission information is available at http://www.nature.com/reprints
Publisher's note Springer Nature remains neutral with regard to jurisdictional claims in published maps and institutional affiliations.

(c) (i) Open Access This article is licensed under a Creative Commons Attribution 4.0 International License, which permits use, sharing, adaptation, distribution and reproduction in any medium or format, as long as you give appropriate credit to the original author(s) and the source, provide a link to the Creative Commons license, and indicate if changes were made. The images or other third party material in this article are included in the article's Creative Commons license, unless indicated otherwise in a credit line to the material. If material is not included in the article's Creative Commons license and your intended use is not permitted by statutory regulation or exceeds the permitted use, you will need to obtain permission directly from the copyright holder. To view a copy of this license, visit http://creativecommons.org/ licenses/by/4.0/.

(C) The Author(s) 2019 